\title{
WHAT'S THE USE? \\ WeLfaRE Estimates fROM REVEALED \\ Preference Models When Weak COMPLEMENTARITY DOES NOT HOLD
}

Joseph A. Herriges, Catherine L. Kling, and Daniel J. Phaneuf

Working Paper 00-WP 258

November 2000 


\title{
What's the Use? \\ Welfare Estimates from Revealed Preference Models when Weak Complementarity Does Not Hold
}

\author{
Joseph A. Herriges, Catherine L. Kling, and Daniel J. Phaneuf
}

Working Paper 00-WP 258

November 2000

\author{
Center for Agricultural and Rural Development \\ lowa State University \\ Ames, lowa 50011-1070 \\ www.card.lastate.edu
}

Joseph A. Herriges and Catherine L. Kling are professors, Department of Economics, lowa State University. Daniel J. Phaneuf is an assistant professor, Department of Agricultural and Resource Economics, North Carolina State University.

The authors would like to acknowledge the many helpful comments and suggestions made by Ted McConnell and Kerry Smith.

This research was supported in part by the U.S. Environmental Protection Agency and by the Western Regional Research project W-133. Although the research described in this article has been funded in part by the United States Environmental Protection Agency through R82-5310-010 to the authors, it has not been subject to the Agency's required peer review policy and therefore does not necessarily reflect the views of the Agency and no official endorsement should be inferred. All remaining errors are, of course, our own.

This paper is available on the CARD website: www.card.iastate.edu. Permission is granted to reproduce this information with appropriate attribution to the authors and the Center for Agricultural and Rural Development, lowa State University, Ames, lowa 50011-1070.

For questions and comments about the contents of this paper, please contact: Joseph A. Herriges, Department of Economics, 260 Heady Hall, lowa State University, Ames, lowa 50011-1070. Email: iaherria@iastate.edu.

lowa State University does not discriminate on the basis of race, color, age, religion, national origin, sexual orientation, sex, marital status, disability, or status as a U.S. Vietnam Era Veteran. Any persons having inquiries concerning this may contact the Director of Affirmative Action, 318 Beardshear Hall, 515-294-7612. 


\begin{abstract}
The focal point of the revealed preference (RP) valuation literature, including recreation demand and random utility maximization (RUM) models, has been on eliciting the "use" value associated with environmental amenities; i.e., that portion of value associated with direct use of a resource. Mäler's (1974) concept of weak complementarity is typically invoked to justify this focus. Indeed, weak complementarity explicitly or implicitly underlies most of the RP literature. In this paper, we consider the measurement of welfare in RP models when weak complementarity does not hold. In particular, the Kuhn-Tucker (KT) framework (e.g., Phaneuf et al. 2000) does not impose weak complementarity a priori, raising the possibility of rejecting weakly complementary in estimation and the question as to what is the proper welfare measure to report. Although existence value cannot be measured, we argue that in some circumstances there are components of total value outside of use value onto which RP methods may be able to shed light.
\end{abstract}

Keywords: Revealed preference, valuation, weak complementarity, use value 



\section{WHAT'S THE USE? WELFARE ESTIMATES FROM REVEALED PREFERENCE MODELS WHEN WEAK COMPLEMENTARITY DOES NOT HOLD}

\section{Introduction}

A large literature on the valuation of environmental quality changes based upon behavioral data on use of the environment has developed over the past 30 years. These models have been variously referred to as recreation demand, travel cost, and/or revealed preference (RP) models and have employed a variety of demand and/or random utility estimation techniques. The purpose of these models has been, almost without exception, to estimate the value of the direct use of these resources. Thus, the surplus measures estimated have often been referred to as "use" values.

Mäler's (1974) concept of weak complementarity has typically been invoked to justify the focus on use values explicitly or implicitly underlying most of the revealed preference literature. In brief, the property of weak complementarity implies that if an individual does not directly use an environmental good, he or she places no value on changes in the quality attributes of that good. Hence, there is no value associated with environmental quality except that which accrues from using the good. Most discussions of weak complementarity begin and end with comments along these lines. Thus, although numerous RP models invoke weak complementarity, few papers give serious attention to empirical specification of RP models or their interpretation in its absence.

The purpose of this paper is to consider the measurement of welfare from RP models in which weak complementarity may not hold. Specifically, the Kuhn-Tucker (KT) framework of Wales and Woodland (1983) has recently been applied (e.g., Phaneuf and Herriges 1999; Phaneuf et al. 2000) in modeling recreation demand, yet within this framework the analyst need not impose a priori weak complementarity in the functional form for preferences. This raises both the possibility of rejecting the weak complementarity assumption in estimation and the 
question as to what components of value are revealed by the non-weakly complementary model. It is important that we note at the outset that pure "existence value" (as we will define it later) simply cannot be estimated from RP data. This well-understood point is not in contention here. Rather, we are simply interested in what complications arise to computing welfare estimates from RP models in the absence of weak complementarity. For example, if weak complementarity does not hold, what is the appropriate interpretation of the traditionally computed welfare measures (i.e., areas under the estimated demand curve)? Does the lack of weak complementarity bias the estimate of this value? What interpretation might the analyst give to the residual value that is present even when demand is zero? Should the welfare analyst impose weak complementarity for estimation purposes even when he or she suspects its absence?

The remainder of the paper is divided into four sections. We begin in Section 2 by developing a formal decomposition of total value based upon Hanemann (1988), emphasizing those components of value that are exposed by revealed preference data when the weak complementarity does not hold. Section 3 then outlines the KT modeling framework. We describe the model with and without weak complementarity and the range of competing welfare measures that one can compute when weak complementarity is not imposed. The choice among these welfare measures depends in large part upon the perceived source of the violation of weak complementarity. Thus, in Section 4 we explore three competing rationales for observing violations of weak complementarity and their respective implications for welfare analysis. These issues are then explored empirically in Section 5 using data from a survey on wetland usage in the state of Iowa. Six thousand residents were sampled in the spring of 1998, providing data on the number of visits they took to wetland areas in the state and the costs of those visits. Using an estimated KT model, we compare and contrast competing measures of the welfare improvements resulting from increased pheasant populations in the state. Finally, Section 6 concludes the paper.

\section{The Components of Value Exposed by Revealed Preference Data}

A number of authors have decomposed total value into use value and existence value associated with changes in environmental quality relying upon weak complementarity to define the two pieces (Madariaga and McConnell [1987]; Freeman [1993] identifies three components 
of value: use value, pure existence value (value placed on environmental quality completely independent of use), and nonuse value (value the individual gets that is related to use, but which does not disappear when the good is not consumed). Hanemann (1988) defines nonuse and use value but employs a definition that does not invoke weak complementarity to distinguish the two. In this paper, we adopt and modify slightly the approach suggested by Hanemann (1988). The main purpose for the decomposition is to aid our understanding of the inherent limitations on the empirical welfare measures that can be extracted from RP data.

The decomposition process begins by specifying a general structure of consumer preferences that will accommodate both traditional use value and the more controversial indirect use and existence value components. Hanemann (1988) assumes that the direct utility function takes the form

$$
U=T[u(\mathbf{x}, q), q]
$$

where $\mathbf{x}$ is a vector of private market goods and $q$ (a scalar) is a public good (e.g., environmental amenity) taken as given by the individual consumer; $T[u, q]$ is increasing in $u$ and $q$; and $u(\mathbf{x}, q)$ is increasing and quasi-concave in $\mathbf{x}$ and $q$. Note that $q$ enters utility in two separate places, in a group with the private goods $(\mathbf{x})$ and separably on its own. Importantly, the marginal rates of substitution between observed consumption bundles (the $x$ 's) will be independent of the second component of the utility function and thus cannot reveal information about the value of changes in that portion of the function. ${ }^{1}$

The corresponding indirect utility function is then given by:

$$
\begin{aligned}
V(\mathbf{p}, q, y) & \equiv \underset{\mathbf{x}}{\operatorname{Max}}\left\{T[u(\mathbf{x}, q), q] \mid \mathbf{p}^{\prime} \mathbf{x} \leq y\right\} \\
& =T\left[\operatorname{Max}\left\{u(\mathbf{x}, q) \mid \mathbf{p}^{\prime} \mathbf{x} \leq y\right\}, q\right] \\
& =T[v(\mathbf{p}, q, y), q]
\end{aligned}
$$

where $v(\mathbf{p}, q, y) \equiv \underset{\mathbf{x}}{\operatorname{Max}}\left\{u(\mathbf{x}, q) \mid \mathbf{p}^{\prime} \mathbf{x} \leq y\right\}$. Again, as the second line of equation (2) emphasizes, all of the interactions between the consumer's activity in the marketplace (including recreational demand) and the public good $q$ are revealed through $v(\mathbf{p}, q, y)$ and independent of the form of $T(\cdot, q)$. Consequently, RP data simply cannot be used to estimate the form of $T(\cdot, q)$. 
Finally, we can specify the corresponding expenditure function as:

$$
\begin{aligned}
E(\mathbf{p}, q, \tilde{U}) & \equiv \operatorname{Min}_{\mathbf{x}}\left\{\mathbf{p}^{\prime} \mathbf{x} \mid T[u(\mathbf{x}, q), q] \geq \tilde{U}\right\} \\
& =\underset{\mathbf{x}}{\operatorname{Min}}\left\{\mathbf{p}^{\prime} \mathbf{x} \mid u(\mathbf{x}, q) \geq \tilde{u}(\tilde{U}, q)\right\} \\
& =e[\mathbf{p}, q, \tilde{u}(\tilde{U}, q)]
\end{aligned}
$$

where $\tilde{u}(\tilde{U}, q)$ is defined implicitly by $T[\tilde{u}, q]=\tilde{U}$ and $e(\mathbf{p}, q, \tilde{u}) \equiv \underset{\mathbf{x}}{\operatorname{Min}}\left\{\mathbf{p}^{\prime} \mathbf{x} \mid u(\mathbf{x}, q) \geq \tilde{u}\right\}$ denotes the standard expenditure function. ${ }^{2}$ Note that the utility level at which the traditional expenditure function is evaluated is adjusted for the second role of $q$ in preferences and that generally $E(\mathbf{p}, q, \tilde{u}) \neq e(\mathbf{p}, q, \tilde{u})$.

Turning to welfare valuations, it is natural to define the total compensating variation $\left(C^{T}\right)$ for a change in the level of the resource from $q^{0}$ to $q^{1}$ as

$$
T\left[v\left(\mathbf{p}^{0}, q^{0}, y\right), q^{0}\right]=T\left[v\left(\mathbf{p}^{0}, q^{1}, y-C^{T}\right), q^{1}\right] .
$$

Hanemann (1988) suggests the following decomposition:

$$
C^{T}=C^{R}+\tilde{C}
$$

where $C^{R}$ is implicitly defined by

$$
T\left[v\left(\mathbf{p}^{0}, q^{0}, y\right), q^{0}\right]=T\left[v\left(\mathbf{p}^{0}, q^{1}, y-C^{R}\right), q^{0}\right]
$$

and $\tilde{C}$ satisfies

$$
T\left[v\left(\mathbf{p}^{0}, q^{0}, y\right), q^{0}\right]=T\left[v\left(\mathbf{p}^{0}, q^{0}, y-\tilde{C}\right), q^{1}\right] .
$$

Notice that $C^{R}$ compensates for the impact that the change in $q$ has on the first argument in $T(\cdot, \cdot)$, whereas $\tilde{C}$ compensates for the impact of the change on the second argument of $T(\cdot, \cdot)$. Unfortunately, the decomposition in equation 5) holds only if the marginal utility of income is constant. ${ }^{3}$ However, the following modified version of equation 5 ) can be used:

$$
C^{T}=C^{R}+C^{E}
$$

where $C^{E}$ is implicitly defined by

$$
T\left[v\left(\mathbf{p}^{0}, q^{1}, y^{R}\right), q^{0}\right]=T\left[v\left(\mathbf{p}^{0}, q^{1}, y^{R}-C^{E}\right), q^{1}\right]
$$

and $y^{R} \equiv y-C^{R}$. Comparing equations (4), (6) and (9), it is clear that what we have is a sequential compensation for the change in $q$, with: 


$$
\begin{aligned}
T\left[v\left(\mathbf{p}^{0}, q^{0}, y\right), q^{0}\right] & =T\left[v\left(\mathbf{p}^{0}, q^{1}, y-C^{R}\right), q^{0}\right] \\
& =T\left[v\left(\mathbf{p}^{0}, q^{1}, y-C^{R}-C^{E}\right), q^{1}\right] .
\end{aligned}
$$

The compensation $C^{R}$ in the first line of equation (10) is used to offset the impact that the change in $q$ has on $v(\mathbf{p}, q, y)$. In contrast, $C^{E}$ denotes the additional compensation required to make the individual whole; i.e., compensating for the impact that the change in $q$ has on the second argument in $T(\cdot, q)$ given $C^{R}$ has already been paid. ${ }^{4}$ Throughout the remainder of the paper, we refer to $C^{R}$ as the revealable portion of compensation and $C^{E}$ as existence value. ${ }^{5}$ Note that this definition of existence value makes no use of the property of weak complementarity (as others have often done in the definition of existence value). It is worth reemphasizing one final time that this piece of value cannot be recovered from RP data. This inherent limitation of RP is well known and will receive no further attention here. ${ }^{6}$

Rather, our focus will turn to the remaining piece, which we have termed $C^{R}$. This piece is of particular interest because it describes the portion of preferences about which we can potentially infer something from RP data. The next question is how much of $C^{R}$ can be inferred from RP data. This is where Mäler's (1974) concept of weak complementarity becomes helpful. We note that $C^{R}$ can itself be decomposed into pieces as follows:

$$
C^{R}=C^{U}+C^{I U}
$$

with $C^{I U}$ implicitly defined by

$$
v\left[\tilde{\mathbf{p}}\left(q^{0}\right), q^{0}, y\right]=v\left[\tilde{\mathbf{p}}\left(q^{1}\right), q^{1}, y-C^{I U}\right],
$$

where $\tilde{\mathbf{p}}(q)$ denotes the price vector at which $\mathbf{x}=\mathbf{0}$ and $C^{U} \equiv C^{R}-C^{I U}$. It seems intuitive to refer to $C^{I U}$ as the "indirect use" value, as it represents welfare changes when the associated market goods are not in use, whereas $C^{U}$ corresponds to direct "use" value. ${ }^{7}$ The components $C^{U}$ and $C^{I U}$ can be equivalently defined in terms of the expenditure function as: ${ }^{8}$

$$
C^{U}=\left\{e\left[\mathbf{p}, q^{0}, u^{0}\right]-e\left[\tilde{\mathbf{p}}\left(q^{0}\right), q^{0}, u^{0}\right]\right\}-\left\{e\left[\mathbf{p}, q^{1}, u^{0}\right]-e\left[\tilde{\mathbf{p}}\left(q^{1}\right), q^{1}, u^{0}\right]\right\}
$$

and

$$
C^{I U}=e\left[\tilde{\mathbf{p}}\left(q^{0}\right), q^{0}, u^{0}\right]-e\left[\tilde{\mathbf{p}}\left(q^{1}\right), q^{1}, u^{0}\right]
$$


where $u^{0}=v\left(\mathbf{p}, q^{0}, y\right)$. Mäler's (1974) assumption of weak complementarity recognizes that if

$$
v\left[\tilde{\mathbf{p}}\left(q^{0}\right), q^{0}, y\right]=v\left[\tilde{\mathbf{p}}\left(q^{1}\right), q^{1}, y\right]
$$

then $C^{I U}=0$ and $C^{U}=C^{R}$, capturing all of the revealable value resulting from a change in $q$. Further, $C^{U}$ can be represented as areas under Hicksian demands

$$
C^{U}=\int_{p_{1}^{0}}^{\tilde{p}_{1}\left(q^{1}\right)} x_{1}\left(p_{1}, \mathbf{p}_{-1}, q^{1}, u^{0}\right) d p_{1}-\int_{p_{1}^{0}}^{\tilde{p}_{1}\left(q^{0}\right)} x_{1}\left(p_{1}, \mathbf{p}_{-1}, q^{0}, u^{0}\right) d p_{1},
$$

where $\mathbf{p}_{-1}$ represents the vector of all prices except for good 1 and $x_{1}\left(p_{1}, \mathbf{p}_{-1}, q^{1}, u^{0}\right)$ denotes the Hicksian demand for good 1 . This is a very handy result for applied welfare analysts as it means that once a demand function is estimated, the welfare change associated with a change in quality can be computed via McConnell (1983). ${ }^{9}$ There is no need to explicitly specify the underlying expenditure or indirect utility functions.

As noted previously, the environmental literature has relied almost exclusively upon the assumption of weak complementarity to justify, explicitly or implicitly, welfare valuations extracted from RPs. Indeed, weak complementarity is implicit in the standard random utility maximization (or RUM) model used extensively in the recreation demand literature. This widespread use of the RUM model begs the question as to whether weak complementarity accurately reflects consumer preferences and whether its imposition biases the implied welfare calculations. In contrast, the KT model provides a utility theoretic framework allowing a more general representation of preferences, within which weak complementarity need not be assumed a priori. At the same time, however, it raises that practical issue as to what the appropriate welfare measure should be. In the next section, we outline the KT framework both in terms of the required estimation procedure and the calculation of $C^{R}, C^{U}$, and $C^{I U}$.

\section{The Kuhn-Tucker Framework}

The KT model adopts a top-down specification for preferences, beginning with maximization of the consumer's direct utility function subject to income and nonnegativity constraints. The first-order conditions, given the potential for nonconsumption of a subset of the goods, take the form of the KT conditions. Formally the consumer solves the problem 
$\underset{\mathbf{x}, z}{\operatorname{Max}} u(\mathbf{x}, z, \mathbf{q}, \gamma, \mathcal{\varepsilon})$

subject to

$$
\mathbf{p}^{\prime} \mathbf{x}+z \leq y
$$

and

$$
z \geq 0, x_{j} \geq 0, j=1, \ldots, M
$$

where $u(\cdot)$ is assumed to be a quasi-concave, increasing, and continuously differentiable function of $(\mathbf{x}, z), \mathbf{x}=\left(x_{1}, \ldots, x_{M}\right)^{\prime}$ is a vector of goods to be analyzed (recreation trips), $\mathrm{z}$ is the numeraire good, $\mathbf{p}=\left(p_{1}, \ldots, p_{M}\right)^{\prime}$ is a vector of commodity prices (travel costs), $\mathbf{q}=\left(q_{1}, \ldots, q_{M}\right)^{\prime}$ is a vector of site-specific quality attributes, $y$ denotes annual income, $\gamma$ is a vector of parameters to be estimated, and $\varepsilon=\left(\varepsilon_{1}, \ldots, \varepsilon_{M}\right)^{\prime}$ is a vector of random disturbances capturing the variation in preferences in the population. Note that a priori, the top-level specification of utility need not in general exhibit the property of weak complementarity.

Assuming the numeraire good is necessary, the first-order conditions for this problem are given by

$$
u_{j} \leq p_{j} u_{z} ; x_{j} \geq 0 ; x_{j}\left[u_{j}-p_{j} u_{z}\right]=0, j=1, \ldots, M .
$$

Given assumptions on the structure of the utility function, the KT conditions can be rewritten as

$$
\varepsilon_{j} \leq g_{j}(\mathbf{x}, y, \mathbf{q}, \gamma) ; \quad x_{j} \geq 0 ; x_{j}\left[\varepsilon_{j}-g_{j}(\mathbf{x}, y, \mathbf{q}, \gamma)\right]=0, \quad j=1, \ldots, M,
$$

where $g_{j}(\cdot)$ is a function of observed variables and parameters to be estimated, determined by the choice of functional form for utility. Equation (21) provides the basis for forming estimating equations for the model. Given a distribution for the error terms, the probability of observing each individual's outcome in the data can be determined from equation 21) and maximum likelihood used to recover estimates of the parameters. ${ }^{10}$

Because of the nonnegativity constraints, the demand system, and hence the indirect utility function of interest for welfare analysis, is nondifferentiable. For example, if there are $M$ sites available, there are $2^{M}$ different combinations of sites that can be visited, including the possibility of not visiting any of the sites during the season. Therefore there are an equal number of potential demand systems, conditional on the demand regime. Let 


$$
\Omega=\{\varnothing,\{1\},\{2\}, \ldots,\{M\},\{1,2\}, \ldots,\{1, M\}, \ldots,\{1,2, \ldots M\}\}
$$

denote the collection of all possible demand patterns and $v\left(\mathbf{p}_{\omega}, \mathbf{q}, y, \gamma, \varepsilon\right)$ denote the indirect utility function when the individual is restricted to the commodities indexed by $\omega \in \Omega$. Note the maximization process implies the conditional indirect utility function is a function of only the prices of consumed goods, whereas the absence of weak complementarity implies the conditional indirect utility function may in fact be a function of all quality attributes, regardless if they are consumed. The individual's unconditional indirect utility function is then given by

$$
v(\mathbf{p}, \mathbf{q}, y, \gamma, \varepsilon)=\max _{\omega \in \Omega}\left\{v_{\omega}\left(\mathbf{p}_{\omega}, \mathbf{q}, y, \gamma, \varepsilon\right)\right\} .
$$

This corresponds to the indirect utility function, $v(\mathbf{p}, \mathbf{q}, y)$, which is in fact the separable component of the overall utility function given in equation (2). As noted above, RP methods can at best expose information on resource values given by $C^{R}$. In the particular case of the KT model, this is implicitly defined by

$$
\max _{\omega \in \Omega^{0}}\left\{v_{\omega}\left(\mathbf{p}_{\omega}, \mathbf{q}^{0}, y, \gamma, \mathcal{\varepsilon}\right)\right\}=\max _{\omega \in \Omega^{1}}\left\{v_{\omega}\left(\mathbf{p}_{\omega}, \mathbf{q}^{1}, y-C^{R}, \gamma, \varepsilon\right)\right\}
$$

and may, if preferences are not weakly complementary, include indirect use value. Direct use value, or $C^{U}$ as defined in equation (13), is given by $C^{U}=C^{R}-C^{I U}$, where $C^{I U}$ is implicitly defined by

$$
\max _{\omega \in \Omega^{0}}\left\{v_{\omega}\left(\tilde{\mathbf{p}}_{\omega}, \mathbf{q}^{0}, y, \gamma, \varepsilon\right)\right\}=\max _{\omega \in \Omega^{1}}\left\{v_{\omega}\left(\tilde{\mathbf{p}}_{\omega}, \mathbf{q}^{1}, y-C^{I U}, \gamma, \varepsilon\right)\right\},
$$

where $\tilde{\mathbf{p}}_{\omega}$ is the vector of choke prices for each of the demand regimes. Preferences in equation (17) are characterized via estimation up to an unobserved vector of error terms, and no closed form for the compensating surpluses given by equations 24) and 25) exists. Given an estimated distribution for the error term, however, Monte Carlo integration can be used to obtain estimates of the expected value of the surplus measures. ${ }^{11}$

Having described the top-down approach of the KT model, we pause momentarily to mention a second, fundamentally different approach to recovering estimates of consumer preferences based on a bottom-up approach suggested by Hausman (1981). This strategy begins with the specification and estimation of ordinary demand equations, from which the quasi expenditure and indirect utility functions can be recovered via integration up to a constant of integration. For purposes of welfare measurement over price changes, the quasi expenditure 
function is sufficient to calculate compensating variation. Welfare measures of quality changes are problematic, however, because the constant of integration will in general depend on quality measures. Larson (1991) suggests solving this by introducing additional information into the integration problem: namely, the weak complementarity condition on the expenditure function stating that the change in expenditure for a change in quality, when evaluated at the choke price, must be zero. With this additional condition, Larson (1991) shows it is possible to recover a quasi-indirect and weakly complementary utility function up to a constant of integration from estimates of any system of ordinary demand equations, from which the use-value welfare effects of quality changes can be evaluated. Larson's (1991) suggestion provides the economist with a useful tool for estimating weakly complementary preferences if it is thought that this is a reasonable restriction on preferences. However, it does not provide guidance as to whether or not weak complementarity should be imposed a priori and, if not, what is the proper welfare measure? Furthermore, recovering a characterization of preferences via the bottom-up approach is much more difficult in the presence of corner solutions, because regime-specific, quasiindirect utility functions must be recovered from the demand system specification. More importantly, information as contained in equation (20) is typically not available for the construction of utility-theoretic, endogenous-regime-switching conditions. In the presence of binding nonnegativity constraints it is therefore likely that the top-down approach of the KT model has a significant comparative advantage.

Returning, then, to discussion of the KT model, its estimation requires specification of the functional form for utility and distribution of the error terms. Given the complexities of estimation and welfare calculations, currently only relatively simple functional forms have been used. $^{12}$ In the application that follows we assume utility is given by a version of the LES utility function such that

$$
u(\mathbf{x}, z ; \mathbf{q}, \boldsymbol{\gamma}, \boldsymbol{\varepsilon})=\sum_{j=1}^{M} \Psi_{j}\left(q_{j}, \varepsilon_{j}\right) \ln \left(x_{j}+\theta_{j}\right)+\ln (z)
$$

where $\gamma=(\delta, \theta), \Psi_{j}$ is a quality index given by $\Psi_{j}\left(q_{j}, \varepsilon_{j}\right)=\exp \left(\delta_{0}+\delta_{1} q_{j}+\varepsilon_{j}\right)$, and $q_{j}$ is a site quality variable. This choice allows straightforward derivations of the estimating equations given in equation 21) and provides for tractable welfare calculations. There is a large literature concerned with the linear expenditure system with much of it focusing on the interpretation that 
is given to the $\theta_{j}$ parameters. The original demand work employing the LES utility function interpreted $-\theta_{j}$ as a "subsistence quantity" for which no utility is obtained. Given subsistence expenditures, "supernumerary income" is then allocated among the available goods. This interpretation requires that $\theta_{j}<0$, which in turn implies strong and unreasonable restrictions on the price and income elasticities that can be recovered from the model (see, e.g., King 1979). However, more recent literature concerning the LES (e.g. Green and Hassan 1980; Pollak and Wales 1992) has demonstrated that the $\theta_{j}{ }^{\prime} s$ need not be restricted in sign in order to provide a consistent representation of preferences. Furthermore, additional flexibility in elasticity values is possible when the signs of these parameters are allowed to be positive.

This is important for our purposes in that in the presence of corner solutions the utility function is only defined for $\theta_{j}>0$. Furthermore, as Larson (1991, p. 103) notes, preferences exhibit weak complementarity between $x_{j}$ and $q_{j}$ for the special case of $\theta_{j}=1$, whereas for $\theta_{j} \neq 1$ weak complementarity does not hold. Thus, given this specification of utility, estimation may lead to a recovery of preferences for which weak complementarity does not hold, implying the computation of $C^{R}$ from equation (16) will not be equal to $C^{U}$. The analyst is then faced with a decision on the proper welfare measure to report. In the following section we discuss several explanations for why weak complementarity may fail to hold and ramifications for which welfare measure should be reported.

\section{Rationale for Observing Violations of Weak Complementarity}

We suggest three possible explanations for the estimation of non-weakly complementary preferences. First, there may be one or more goods that form the set of goods that are weakly complementary to $q$. Madariaga and McConnell (1987) consider this possibility when they note that their definition of existence value includes off-site use values. Bockstael and Kling (1988) derive the appropriate welfare measures if all of the weakly complementary demands are estimated and used for welfare computation. If the analyst has included only one of the goods in the empirical model, the omitted variables may show up as a rejection of weak complementarity. Second, the absence of weak complementarity may be a direct result of the individual's 
preference for environmental quality. For example, in the context of the household production framework, environmental quality may be an essential good in the production of environmental services, whereas $x$ may not be (see, e.g., Bockstael and McConnell 1983). Third and finally, estimation of preferences that appear inconsistent with weak complementarity may stem from econometric problems such as model specification and/or measurement errors. In this section, we discuss each of these explanations in turn. We consider their implications for specifying empirical models of RPs as well as the appropriate computation and interpretation of welfare measures coming from such models.

\section{Weak Complementarity with Sets of Goods-An Omitted Variables Story}

Bockstael and Kling (1988) derive welfare measures for changes in environmental quality when quality is weakly complementary to a set of goods. Suppose there are two goods that are weak complements to $q, x_{1}$ and $x_{2}$. This means that when both $x_{1}$ and $x_{2}$ equal zero, the marginal utility of $q$ also is zero. Bockstael and Kling (1988) demonstrate that in this case, the correct welfare measure for a change in $q$ can be written as the sum of areas under demand curves $^{13}$

$$
\begin{aligned}
C^{R} & =C^{U} \\
& =\left[\int_{p_{1}^{0}}^{\tilde{p}_{1}\left(q^{1}\right)} x_{1}\left(p_{1}, p_{2}^{0}, q^{1}, u^{0}\right) d p_{1}-\int_{p_{1}^{0}}^{\tilde{p}_{1}\left(q^{0}\right)} x_{1}\left(p_{1}, p_{2}^{0}, q^{0}, u^{0}\right) d p_{1}\right] \\
& +\left[\int_{p_{2}^{0}}^{\tilde{p}_{2}\left(q^{1}\right)} x_{2}\left[\tilde{p}_{1}\left(q^{1}\right), p_{2}, q^{1}, u^{0}\right] d p_{2}-\int_{p_{2}^{0}}^{\tilde{p}_{2}\left(q^{0}\right)} x_{2}\left[\tilde{p}_{1}\left(q^{0}\right), p_{2}, q^{0}, u^{0}\right] d p_{2}\right] \\
& =C_{1}^{U}+\tilde{C}_{2}^{U}
\end{aligned}
$$

where

$$
C_{1}^{U}=\int_{p_{1}^{0}}^{\tilde{p}_{1}\left(q^{1}\right)} x_{1}\left(p_{1}, p_{2}^{0}, q^{1}, u^{0}\right) d p_{1}-\int_{p_{1}^{0}}^{\tilde{p}_{1}\left(q^{0}\right)} x_{1}\left(p_{1}, p_{2}^{0}, q^{0}, u^{0}\right) d p_{1}
$$

and

$$
\tilde{C}_{2}^{U}=\int_{p_{2}^{0}}^{\tilde{p}_{2}\left(q^{1}\right)} x_{2}\left[\tilde{p}_{1}\left(q^{1}\right), p_{2}, q^{1}, u^{0}\right] d p_{2}-\int_{p_{2}^{0}}^{\tilde{p}_{2}\left(q^{0}\right)} x_{2}\left[\tilde{p}_{1}\left(q^{0}\right), p_{2}, q^{0}, u^{0}\right] d p_{2} .
$$


In terms of expenditure functions, $C^{R}$ can be expressed equivalently as

$$
\begin{aligned}
C^{R} & =C^{U} \\
& =e\left(p_{1}^{0}, p_{2}^{0}, q^{0}, u^{0}\right)-e\left(p_{1}^{0}, p_{2}^{0}, q^{1}, u^{0}\right)
\end{aligned}
$$

Our point of departure is to consider what happens when the analyst either does not know or cannot measure $x_{2}$ and thus cannot estimate the second demand function. Further, the analyst cannot include the price of $x_{2}$ in the estimating equation for $x_{1}$, possibly generating biased coefficient estimates for the first demand equation, depending of course on the correlation patterns between the omitted variable and the included ones.

What are the implications of this misspecification for welfare measurement? Obviously, the analyst cannot estimate the sum of areas as represented in equation (27) because $x_{2}$ is unknown. However, the analyst might be able to recover an unbiased estimate of $C_{1}^{U}$ in equation 28).Further, the analyst might be able to use knowledge of the form of the expenditure function underlying the estimated demand model to compute some part of $C^{R}$. We are interested in identifying the circumstances under which $C_{1}^{U}$ and/or $C^{R}$ might be recovered from RP estimates. As suggested earlier, the answer depends on the correlation patterns between the omitted and included variables.

Suppose first that the omitted price of good 2 is not correlated with any of the other independent variables in the first demand equation. If $x_{2}$ represents magazines, books, videos, and/or other nonconsumption items related to $q$, the assumption of complete independence may be quite reasonable (in fact, $p_{2}$ might be nearly constant across the sample). For intuition in considering the econometric implications, suppose the true demands for both goods are linear; i.e.,

$$
x_{i}=\alpha_{i}+\beta_{i} p_{i}+\gamma_{i} p_{j}+\delta_{i} q \quad i=1,2 ; j \neq i .
$$

Standard omitted variables results indicate that the estimation of the demand for $x_{1}$ in this circumstance will result in unbiased coefficient estimates for each of the slope parameters, but a biased constant term with

$$
E\left(\alpha_{1}\right)=\alpha_{1}+\beta_{2} \bar{p}_{2}^{0}
$$

where $\bar{p}_{2}^{0}$ is the sample average price of $x_{2}$. The sign of the bias to the intercept will depend upon the relationship between $x_{1}$ and $x_{2}$ : if they are substitutes, the constant term will be biased upwards; if they are complements, the bias will be downwards. 
What are the implications for welfare measurement? Because each individual's $p_{2}$ is unknown and cannot be included in the estimating equation, the demand equation is conditioned on the sample average $\bar{p}_{2}^{0}$ rather than the individual's actual $p_{2}$. The estimated welfare for each individual can be expressed as

$$
\hat{C}_{1}^{U}=\int_{p_{1}^{0}}^{\tilde{p}_{1}\left(q^{1}\right)} x_{1}\left(p_{1}, \bar{p}_{2}^{0}, q^{1}, u^{0}\right) d p_{1}-\int_{p_{1}^{0}}^{\tilde{p}_{1}\left(q^{0}\right)} x_{1}\left(p_{1}, \bar{p}_{2}^{0}, q^{0}, u^{0}\right) d p_{1},
$$

yielding a bias of

$$
\begin{aligned}
\operatorname{bias}_{1} & =\left[\int_{p_{1}^{0}}^{\tilde{p}_{1}\left(q^{1}\right)} x_{1}\left(p_{1}, \bar{p}_{2}^{0}, q^{1}, u^{0}\right) d p_{1}-\int_{p_{1}^{0}}^{\tilde{p}_{1}\left(q^{0}\right)} x_{1}\left(p_{1}, \bar{p}_{2}^{0}, q^{0}, u^{0}\right) d p_{1}\right] \\
& -\left[\int_{p_{1}^{0}}^{\tilde{p}_{1}\left(q^{1}\right)} x_{1}\left(p_{1}, p_{2}^{0}, q^{1}, u^{0}\right) d p_{1}-\int_{p_{1}^{0}}^{\tilde{p}_{1}\left(q^{0}\right)} x_{1}\left(p_{1}, p_{2}^{0}, q^{0}, u^{0}\right) d p_{1}\right]
\end{aligned}
$$

Unfortunately, the sign of this bias is generally indeterminate both for any individual and when summed over the sample. ${ }^{14}$ However, two conditions under which this bias will be small are clear:

(1) when $p_{2}$ does not vary across the sample then $p_{2}^{0}=\bar{p}_{2}^{0}$ and there is no bias, and

(2) when $\partial x_{1} / \partial p_{2}=0$ or is small, the bias also will be nonexistent or small.

We now turn to the prospects in this case for the estimation of $C^{R}$. Recall that $C^{R}$ can be written as in equation 30) as the difference between two expenditure functions. Are these recoverable? The answer is yes, but like the recovery of $C_{1}^{U}$, they can only be evaluated at the "wrong" value of $p_{2}$. To see why, note that if we know the Hicksian demand (as required to compute $C_{1}^{U}$ ), we can integrate it over price to recover the expenditure function. Like the demand, the expenditure function will be a function of the own price $\left(p_{1}\right), q$, and the sample average of the price of the second good $\left(\bar{p}_{2}^{0}\right)$ (embedded in the parameter estimates). Thus, computation of the difference in expenditure functions evaluated at the current own price and changes in $q$ is equivalent to

$$
\begin{aligned}
\hat{C}^{R} & =\hat{C}^{U} \\
& =e\left(p_{1}^{0}, \bar{p}_{2}^{0}, q^{0}, u^{0}\right)-e\left(p_{1}^{0}, \bar{p}_{2}^{0}, q^{1}, u^{0}\right)
\end{aligned}
$$


yielding a bias of

$$
\operatorname{bias}_{R}=\left[e\left(p_{1}^{0}, \bar{p}_{2}^{0}, q^{0}, u^{0}\right)-e\left(p_{1}^{0}, \bar{p}_{2}^{0}, q^{1}, u^{0}\right)\right]-\left[e\left(p_{1}^{0}, p_{2}^{0}, q^{0}, u^{0}\right)-e\left(p_{1}^{0}, p_{2}^{0}, q^{1}, u^{0}\right)\right]
$$

Unfortunately, the sign and magnitude of this bias is again indeterminate. Under very restrictive conditions one might be able to estimate the correct structure for the expenditure function and obtain unbiased estimates of $C^{U} \cdot{ }^{15}$ However, these conditions are unlikely to hold in practice and we would recommend instead that $\hat{C}_{1}^{U}$ be computed and reported as what it is, a measure of the use value stemming from the combined use of $x_{1}$ and $q$, and acknowledging the missing components $\tilde{C}_{2}^{U}$ and $C^{E}$.

Now consider the case where the omitted price of good 2 is perfectly correlated with one of the variables in the $x_{2}$ equation. A likely candidate would seem to be $p_{1}$. In particular, if $x_{1}$ represents visits to a recreation site for the purpose of fishing and $x_{2}$ represents visits to the same site with the purpose of swimming, hiking, or any other activity, the perfect correlation of prices is quite likely. Following Bockstael and Kling (1988), suppose the prices of the two commodities are related in a linear fashion, so that

$$
p_{2}=\phi_{1}+\phi_{2} p_{1},
$$

where $\phi_{1}$ and $\phi_{2}$ are constants. In this case, estimation of the linear model in (31) omitting $p_{2}$ will yield the following properties of the estimated coefficients

$$
E\left(\hat{\alpha}_{1}\right)=\alpha_{1}+\beta_{2} \phi_{1}
$$

and

$$
E\left(\hat{\beta}_{1}\right)=\beta_{1}+\beta_{2} \phi_{2} .
$$

In this case, the relationship between $p_{1}$ and $p_{2}$ is embedded in the estimated demand function for $x_{1}$ since the estimated coefficients incorporate the relationship. What does this mean for the recovery of $C_{1}^{U}$ and $C^{R}=C^{U}$ ? As Bockstael and Kling [1988, p. 660] note in a similar situation, by varying $p_{1}$, we are implicitly varying $p_{2}$ as well. Thus, when constructing $C_{1}^{U}$ using equation (28), one is in fact computing

$$
\widehat{C}_{1}^{U}=\int_{P} x_{1}\left(p_{1}, p_{2}, q^{1}, u^{0}\right) d t-\int_{P} x_{1}\left(p_{1}, p_{2}, q^{0}, u^{0}\right) d t
$$


where the price path $\mathrm{P}$ corresponds to $p_{1}=t$ and $p_{2}=\phi_{1}+\phi_{2} t$. The remaining component of $C^{R}=C^{U}$ corresponds to

$$
\begin{aligned}
\widehat{C}_{2}^{U} & =C^{U}-\hat{C}_{1}^{U} \\
& =\int_{P} x_{2}\left(p_{1}, p_{2}, q^{1}, u^{0}\right) d t-\int_{P} x_{2}\left(p_{1}, p_{2}, q^{0}, u^{0}\right) d t .
\end{aligned}
$$

Again, without strong structural assumptions regarding the nature of preferences, the analyst is unlikely to have an estimate of the functional form for $x_{2}$, which in turn precludes the computation of $\widehat{C}_{2}^{U}$, leaving the $\widehat{C}_{1}^{U}$ as that portion of $C^{R}$ that is recoverable.

\section{Environmental Quality as an Essential Good-A Household Production Story}

An alternative explanation for violations of weak complementarity arises from the household production approach to consumer behavior. ${ }^{16}$ In this case, individuals are assumed hold preferences over a bundle of commodities $\mathbf{z}=\left(z_{1}, \ldots, z_{M}\right)$. These commodities are in turn produced by combining the market commodities (x) and the public good $(q)$ through the household production process $t(\mathbf{z}, \mathbf{x}, q)=0$. If the production technology follows the simpler structure with $\mathbf{z}=\mathbf{z}(\mathbf{x}, q)$, then consumer preferences take the form

$$
u=u[\mathbf{z}(\mathbf{x}, q)] .
$$

As Freeman (1993, p. 149) notes, weak complementarity in this context corresponds to the assumption that $\mathbf{x}$ is an essential input to the production of the $z_{j}$ 's. However, this need not be the case. Indeed, if $\mathbf{x}$ is not an essential input and $q$ is, then weak complementarity does not hold and there is more to $C^{R}$ than the "use" value associated with the $x_{i}$ 's. Under this interpretation, there is an intrinsic value to the public good not captured by its association with the market. Thus, even when $\mathbf{x}$ is not consumed, changes to the public good alter consumer welfare. ${ }^{17}$

What distinguishes this from the previous case is that there are no omitted variables or other misspecifications in the empirical model. Thus, the model the analyst is estimating is in fact the true model. Consequently, the welfare measure $C^{R}$ derived from equation 6 s fully revealable in this case. 


\section{Specification Errors as the Source of Empirical Violations}

We complete this section by noting one additional explanation for violations of weak complementarity. The previous two explanations have been based on behavioral underpinnings. An alternative explanation may lie with econometric problems. As Randall (1994) has pointed out, prices in RP models are likely measured with error, as are other variables entering the model that depend on an individual's recall while responding to a survey. Thus, it may in fact be that weak complementarity holds, but we reject this in preference estimation due to data problems. Specifically in the case of the LES model, if trip data (i.e., the $x_{i}$ 's) are systematically under- or overstated due to recall errors, these errors are likely to be captured by the estimated $\theta_{i}$ 's in equation (26). which are in turn used to test for violations of weak complementarity. Alternatively, it may be that the utility function we estimate is incorrect or not sufficiently flexible and we reject weak complementarity although it would hold for the individual's true preference function.

Although these explanations may of course be true and undoubtedly contribute somewhat to deviations from weak complementarity, they could in fact be said about any empirical welfare measurement, regardless of whether it is related strictly to use, existence, or some form of indirect use. Hypothesis tests and imputed welfare measures are always conditional upon the underlying model specification. This suggests that caution is appropriate when violations of weak complementarity are found in an LES model and that further research is needed into the use of more flexible functional forms within the KT framework. However, while acknowledging the potential for misspecification errors, we believe that this explanation begs the question of the proper course of action when the chosen specification is not consistent with weak complementarity. The LES model may indeed be an accurate representation of preferences and the available data may indeed be accurate. The question in this case is how should the analyst proceed. In the following section we investigate the various welfare measures discussed above, conditional on any data and/or specification problems that may be present.

\section{Empirical Investigation}

Our empirical investigation centers around data obtained from the 1997 Iowa Wetlands Survey conducted at Iowa State University. The purpose of this survey was to obtain information 
on Iowans' use of wetlands in the state as well as attitudes towards wetlands preservation and conservation. A survey of 6,000 Iowa households was drawn from the general population and from state hunting and fishing license holders, from which 3,131 useable surveys were returned. As part of the survey each individual was given a map of the state, divided into fifteen zones, and asked to record the number of visits to wetlands made to each of the zones during 1997.

Of particular interest for this example are the responses of individuals living in the northcentral part of the state encompassing three of the zones. This area is known as the Des Moines lobe of the North American prairie pothole region. The prairie pothole region is a large, fairly unique section of the continent, including parts of Iowa, Minnesota, the Dakotas, and the Canadian plains provinces. The area is dotted with indentations (formed by retreating glaciers) in otherwise flat landscapes, which are wet for at least part of the year. This type of wetland is ideal habitat for many types of wildlife, including ducks and pheasants, and is important at both the local and continental level. At the local level, these wetlands and the surrounding upland areas provide opportunities for outdoor recreation, including hunting, hiking, and wildlife viewing. In this application we model visits to the three prairie pothole zones by individuals living in the region. This subsample includes 296 respondents, of whom 191 visited a wetland in the region during 1997. Of these individuals, only eleven visited wetlands in each of the three zones comprising the prairie pothole region. The KT model as discussed in section 3 is an attractive model for this application, because it readily accounts for nonparticipation and corner solutions in the data.

Prices of visits to the three sites included in the model were calculated in the typical manner, using round-trip travel distance and time as computed via the software package PCMiler and valuing travel and money costs at $\$ 0.21 /$ mile and one-third the wage rate, respectively. Quality variables enter the model in the form of county-level roadside pheasant counts provided by the Iowa Department of Natural Resources, aggregated to correspond to the three zones included in the survey instrument. For estimation purposes, an effective pheasant count variable is constructed by weighting actual pheasant counts by a dummy variable equal to one if the survey respondent indicated possession of a hunting or fishing license. It is further assumed that the random terms are distributed independent, identical extreme value. While more general distributions are possible (see Phaneuf et al. 2000), this specification provides a closed form for 
the likelihood function and allows direct resampling from the estimated error distribution, significantly simplifying the estimation and welfare calculation process. ${ }^{18}$ Given these assumptions, we estimate the three-site KT model using the prairie pothole subsample from the Iowa Wetlands data, with results of estimation presented in Table 1.

Table 1: Estimation and welfare results

\begin{tabular}{|c|c|c|c|}
\hline \multirow[t]{2}{*}{ Parameters } & \multicolumn{3}{|c|}{ Model } \\
\hline & Unrestricted & Restricted & Logit \\
\hline$\theta_{1}$ & $\begin{array}{c}7.15 \\
(0.83)\end{array}$ & NA & $\mathrm{NA}$ \\
\hline$\theta_{2}$ & $\begin{array}{c}6.72 \\
(0.79)\end{array}$ & NA & NA \\
\hline$\theta_{3}$ & $\begin{array}{c}8.44 \\
(1.00)\end{array}$ & NA & NA \\
\hline$\delta_{0}$ & $\begin{array}{l}-5.78 \\
(0.16)\end{array}$ & $\begin{array}{l}-8.91 \\
(0.25)\end{array}$ & NA \\
\hline$\delta_{1}$ & $\begin{array}{c}0.0103 \\
(0.0021)\end{array}$ & $\begin{array}{c}0.024 \\
(0.0049)\end{array}$ & NA \\
\hline$v$ & $\begin{array}{l}0.5839 \\
(0.033)\end{array}$ & $\begin{array}{c}1.36 \\
(0.074)\end{array}$ & NA \\
\hline$\beta_{0}$ & NA & NA & $\begin{array}{c}1.69 \\
(0.07)\end{array}$ \\
\hline$\beta_{y}$ & NA & NA & $\begin{array}{c}0.05 \\
(0.001)\end{array}$ \\
\hline$\beta_{p}$ & NA & NA & $\begin{array}{c}0.023 \\
(0.001)\end{array}$ \\
\hline Log-likelihood & -1241 & -1354 & -7202 \\
\hline Welfare Scenario & $C^{U}$ & $C^{R}$ & $C^{R}$ \\
\hline $\begin{array}{l}20 \text { percent increase in } \\
\text { pheasant counts at all site }\end{array}$ & $\begin{array}{ll}\$ 322 & \$ 88 \\
(83) & (33) \\
\end{array}$ & $\begin{array}{l}\$ 778 \\
(166) \\
\end{array}$ & $\begin{array}{l}\$ 36 \\
(11) \\
\end{array}$ \\
\hline
\end{tabular}

Note: Standard errors on welfare measures computed via 200 bootstrap replications. 
We estimate two specifications of the KT model, an unrestricted and a restricted version. The unrestricted model freely estimates $\theta_{j}$ for each site whereas the restricted model restricts each of these parameters to equal to one, imposing weak complementarity on the preference structure. Both models are parsimonious in parameters, and in each case all estimates are significantly different from zero at better than the 1 percent confidence level. As expected, increases in pheasant counts at each site will increase utility and positively affect the demand for trips. These parameters characterize preferences, which can then be used to calculate elasticities and other measures of policy interest.

Of particular interest for the topic of this paper are the estimates of $\theta_{j}$. Note that in each case the estimates are significantly different from one at any reasonable confidence level, and that the restricted model is rejected against the unrestricted model in a likelihood ratio test at the 1 percent significance level. Thus, for this specification of utility in this application, weak complementarity is rejected. Welfare calculations corresponding to equation (24) above will contain not only pure use value, but also indirect use. Therefore the analyst must determine which is the correct measure to report. For this example, we consider the effects of a 20 percent increase in pheasant counts throughout the prairie pothole region and calculate three KT welfare measures, along with the comparable repeated multinomial logit welfare measure. Each of these could be considered correct under various assumptions.

If we adopt the interpretation of the omitted variables story-that weak complementarity is rejected because the model does not explicitly model the demand for goods that also are in the weakly complementary set of goods - then it will be most correct to calculate and report $C^{U}$. The degree to which our estimate of $C^{U}$ is biased will depend, as indicated earlier, on the degree of correlation between the prices, the functional form of demand, and the magnitude of the cross price effect. Although the magnitude of the bias is clearly an empirical question that will vary across applications, we suspect that in most cases it will be small enough not to be a significant cause of concern. Thus, the omitted variables interpretation would suggest that the analyst report a use value of about $\$ 88$ per season for a 20 percent increase in the pheasant population. It is useful to once again note that this value does not consider the change in values of the weakly complementary goods that are not included in the model. 
In contrast, if we believe the model is correctly specified and the rejection of weak complementarity is evidence of the essential nature of $q$, then it would seem most correct to calculate and report the full value of $C^{R}$, including both the pure use value and the residual indirect use value. Thus, the analyst would report that a 20 percent increase in the pheasant population would yield about $\$ 322$ of surplus. The analyst might further break this value down into the two components of use (about \$88) and nonuse (about \$234), but it is not clear that there is any intrinsic value to this division.

If instead the analyst believes that model misspecification or measurement errors yield biased measures of $C^{I U}$, it is unclear how one should proceed. A case could likely be made for imposing weak complementarity as a maintained hypothesis, yielding \$778 in this instance, or using the unrestricted model's estimate of $C^{U}=\$ 88$. A final option, which may be preferable given one's belief that weak complementarity ought to hold but is rejected due to misspecification, would be to apply Larson's (1991) method for imposing weak complementarity on preferences. Further research would be necessary to generalize this for the case of corner solutions, but it is likely feasible nonetheless.

In either of the previous three cases, it will be important for the analyst to clearly identify which welfare measure has been calculated and reported. It is apparent from the large differences in the magnitudes of the welfare measures that this decision has potentially large implications for the outcome of benefit cost comparisons and other uses of welfare numbers.

For comparison purposes, we also estimate a standard four-good repeated multinomial logit model. The repeated multinomial logit model conditional indirect utility functions are given by $V_{j}=\beta_{y}\left(y-p_{j}\right)+\beta_{p} p h_{j}+\varepsilon_{j}, j=1, \ldots, 3$, and $V_{0}=\beta_{0}+\beta_{y} y+\varepsilon_{0}$ for the option of not making a trip. Fifty choice occasions were used and income $(y)$ was calculated as annual income divided by the number of choice occasions. This model is of particular interest in the context of our discussion since it, like the restricted KT model, imposes weak complementarity ex ante. Interestingly, the welfare result from the logit model of $\$ 36$ for $C^{R}=C^{U}$ (see Table 1) is of a comparable order of magnitude to the use value from the unrestricted KT model. In contrast, the use value obtained from the ex ante restricted KT model is $\$ 778$. This could be interpreted as providing some anecdotal support for estimating unrestricted preferences in KT models, rather than ex ante imposing weak complementarity. 


\section{Final Remarks}

In this paper, we investigate the implications of non-weakly complementary preferences for applied welfare analysis using RP data. Although existence value cannot be measured using RP data, there is a component of total value outside of standard use value (deemed "indirect use" value here) for which RP approaches may be able to shed some light. The purpose of this paper has been to highlight this issue and begin to investigate its implications for welfare measurement. Our motivation is pragmatic, in that the recently available KT model does not a priori impose weak complementarity, requiring the analyst to determine which is the correct welfare measure to report. Critical to understanding welfare measurement when weak complementarity does not hold is to first understand and define the relevant components of total value. We do so with a particular focus on identifying the components of total value that are recoverable, at least potentially, from RP data and models.

We propose three explanations for why weak complementarity may be violated in any particular empirical setting: weak complementarity with sets of goods, a household production model with quality as an essential input, and econometric problems. We note that the implications for welfare analysis are quite different in each of the cases and that the proper welfare measure will depend on the judgment of the analyst. In laying out these three interpretations, we do not intend to promote one over any other; rather we seek to provide a framework for discussion, interpretation, and future research. However, we do note that those inherently uneasy about anything beyond direct use value in RP models will likely be most comfortable with the first or third interpretation and the associated welfare measures.

The KT model is a convenient framework for investigating these issues empirically as weak complementarity does not have to be imposed ex ante, but rather can be tested for in the context of the model. In contrast, the standard RUM model implicitly imposes weak complementarity and, in doing so, precludes investigation into the consequences of the restriction. An application of the KT model to wetlands usage in the prairie pothole region of Iowa suggests, in fact, that weak complementarity does not hold in the empirical specification between visits to wetlands and pheasant populations. Further, the alternative interpretations of why weak complementarity does not hold yield welfare magnitudes of sufficient difference to warrant further investigation into these issues. Steps in this direction may include estimating KT models using more general functional forms for utility and/or the error distribution. 



\section{Endnotes}

1. This is a generalization of Freeman's [1993, pp. 123-24] "hopeless" case in which he assumes that $q$ enters only as a strongly separable component of utility.

2. It is assumed that $T(u, q)$ is strictly increasing in $u$.

3. A proof of this is provided in the Appendix.

4. Obviously, one could reverse the order of compensation by defining $\tilde{C}^{R}$ such that:

$$
T\left[v\left(\mathbf{p}^{0}, q^{0}, \tilde{y}\right), q^{1}\right]=T\left[v\left(\mathbf{p}^{0}, q^{1}, \tilde{y}-\tilde{C}^{R}\right), q^{1}\right],
$$

where $\tilde{y} \equiv y-\tilde{C}$. In this case, the decomposition would be $C^{T}=\tilde{C}^{R}+\tilde{C}$ and the counterpart to equation (10) would become

$$
\begin{aligned}
T\left[v\left(\mathbf{p}^{0}, q^{0}, y\right), q^{0}\right] & =T\left[v\left(\mathbf{p}^{0}, q^{0}, y-\tilde{C}\right), q^{1}\right] \\
& =T\left[v\left(\mathbf{p}^{0}, q^{1}, y-\tilde{C}-\tilde{C}^{R}\right), q^{1}\right] .
\end{aligned}
$$

There are two reasons to prefer the decomposition in equation (\$). First, from a practical point of view, because the functional form for $T(\cdot, q)$ can never be identified from RP data, $\tilde{C}$ cannot be computed and, consequently, neither can $\tilde{y}$ or $\tilde{C}^{R}$. Second, even if $\tilde{C}$ were known, as long as $q$ is a normal good $C^{R}>\tilde{C}^{R}$, so that $C^{R}$ represents the largest portion of total value that can be extracted from behavioral data.

5. The compensation $C^{E}$ is similar to the notion of Carson et al. (1999) of "passive-use" value; i.e., ". . . those portions of total value ... that are unobtainable using indirect measurement techniques which rely on observed market behavior." (p. 100). We have chosen not to use the term "passive-use" value, however, to avoid confusion with the notion of indirect use (e.g., reading magazines, etc., concerning a recreation site) that is included in $C^{R}$.

6. See LaFrance (1992) for additional discussion regarding testing of the weak complementarity restriction. 7. In Freeman's (1993) terms $C^{I U}$ could also be referred to as "nonuse" value. We avoid this terminology here to prevent confusion, because in other works "nonuse" value and "existence" value have been used synonymously. As previously noted, in this case $C^{E}$ and $C^{I U}$ have quite different interpretations. 8. In fact, as Hanemann (1988, p. 1) notes, decompositions analogous to equations 13) and (14) are valid for any intermediate prices, although the terminologies of use or nonuse values would be less intuitive. 
9. For the time being, we ignore the problem of income effects in the demand equations.

10. See Phaneuf et al. (2000) for further details on implementing the KT model.

11. See Phaneuf et al. (2000) for a discussion of the necessary algorithm for computing welfare measures in the KT model. This process, while computationally intense, is conceptually simple once the conditional indirect utility functions are recovered.

12. Although in fairness to the KT model it should be noted that most recreation demand studies rely on the restrictive linear-in-income form of the RUM model.

13. The expression in equation (27) depresents one way to write the compensating (or equivalent) variation, based on the path of integration $\left(p_{1}^{0}, p_{2}^{0}\right) \rightarrow\left(\tilde{p}_{1}(q), p_{2}^{0}\right) \rightarrow\left(\tilde{p}_{1}(q), \tilde{p}_{2}(q)\right)$. Of course, the same welfare compensation would result if the alternative path $\left(p_{1}^{0}, p_{2}^{0}\right) \rightarrow\left(p_{1}^{0}, \tilde{p}_{2}(q)\right) \rightarrow\left(\tilde{p}_{1}(q), \tilde{p}_{2}(q)\right)$ had been used, yielding $C^{T}=\tilde{C}_{1}^{U}+C_{2}^{U}$. More generally, the welfare measure can be expressed as a line integral that is path independent for the Hicksian welfare measures. See Bockstael and Kling (1988) for the derivation and discussion.

14. For the linear model in equation (31), t can be shown that bias $_{1}$ is in fact zero on average if: (1) the initial price for good 1 (i.e., $p_{1}^{0}$ ) is the same across all households, (2) the initial price of good $2\left(p_{2}^{0}\right)$ is symmetrically distributed about the population mean $\left(\bar{p}_{2}^{0}\right)$, and (3) all households are users (i.e., $x_{1}>0$ ) before (after) a quality increase (decrease). If condition (3) is violated, bias $_{1}$ will on average be negative (positive) for a quality increase (decrease).

15. Ideally, the analyst knows that the second good exists and will be able to realistically impose sufficient structure on preferences to allow all of the parameters of the expenditure function to be recovered through the estimated demand function for good 1 (e.g., in an LES system). The resulting bias $_{R}$ would then be limited if $p_{2}^{0}$ varied little, if at all, in the population.

16. See, for example, Becker (1965), Lancaster (1966), and Bockstael and McConnell (1993).

17. While difficult to quantify, one can tell stories consistent with these types of preferences. For example, if an individual is unable to go fishing with his friends because of other commitments but later enjoys hearing stories about how great the fishing was, he is producing utility from the environmental good without consuming the complement. Thus, weak complementarity does not hold in the structure of the individual's preferences for the single good.

18. Additional details on estimation and welfare calculation can be found in Phaneuf and Herriges (1999) or Phaneuf et al. (2000). Example GAUSS programs for estimation and welfare measures for the LES/EV model are available from the authors upon request. 


\section{Appendix}

The purpose of this appendix is to demonstrate that the welfare decomposition in equation (5) and originally proposed by Hanemann (1988) holds only if the marginal utility of income is constant. To see this, note that, from equation (@) $C^{R}$ can be equivalently defined as implicitly solving the equality

$$
v\left(\mathbf{p}^{0}, q^{0}, y\right)=v\left(\mathbf{p}^{0}, q^{1}, y-C^{R}\right) .
$$

However, equations (4) and (7) imply that:

$$
\begin{aligned}
T\left[v\left(\mathbf{p}^{0}, q^{0}, \tilde{y}\right), q^{1}\right] & =T\left[v\left(\mathbf{p}^{0}, q^{1}, y-C^{T}\right), q^{1}\right] \\
& =T\left[v\left(\mathbf{p}^{0}, q^{1}, \tilde{y}-C^{R}\right), q^{1}\right],
\end{aligned}
$$

where $\tilde{y} \equiv y-\tilde{C}$. This in turn implies that

$$
v\left(\mathbf{p}^{0}, q^{0}, \tilde{y}\right)=v\left(\mathbf{p}^{0}, q^{1}, \tilde{y}-C^{R}\right) .
$$

Clearly, equations 43) and (45) vill generally hold only if the marginal utility of income is constant. 



\section{References}

Becker, G. S. 1965. “A Theory of the Allocation of Time.” Economic Journal 75: 493-517.

Bockstael, N. E., and C. L. Kling. 1988. "Valuing Environmental Quality: Weak Complementarity with Sets of Goods." American Journal of Agricultural Economics 70: 654-62.

Bockstael, N. E., and K. E. McConnell. 1983. "Welfare Measurement in the Household Production Framework." American Economic Review 73: 806-14. . 1993. "Public Goods as Characteristics of Non-market Commodities." Economics Journal 103: 1244-57.

Carson, R. T., N. E. Flores, and R. C. Mitchell. 1999. "The Theory and Measurement of PassiveUse Value." In Valuing Environmental Preferences: Theory and Practice of the Contingent Valuation Method in the U.S., E.U., and Developing Countries, I. J. Bateman and K. G. Willis, eds. Oxford: Oxford University Press.

Freeman, A. M. 1993. The Measurement of Environmental and Resource Values: Theory and Methods. Resources for the Future, Washington, D.C.

Green, R., and Z. A. Hassan. 1980. "Choices and Consequences: Comment," American Journal of Agricultural Economics 62: 174-75.

Hanemann, W. M. 1988. “Three Approaches to Defining 'Existence' or 'Non-use' Value under Certainty." Unpublished paper, University of California, Berkeley.

Hausman, J. A. 1981. "Exact Consumer's Surplus and Dead Weight Loss." American Economic Review 71: 662-76.

King, R. A. 199. "Choices and Consequences.” American Journal of Agricultural Economics 61: 839-48.

LaFrance, J. 1992. "Incomplete Demand Systems, Weak Separability, and Weak Complementarity." Department of Agricultural and Resource Economics, Working Paper \#77, University of Arizona, Tucson.

Lancaster, K. J. 1966. “A New Approach to Consumer Theory,” Journal of Political Economy, 74: $132-57$. 
Larson, D. M. 1991. "Recovering Weakly Complementary Preferences," Journal of Environmental Economics and Management 21: 97-108.

Madariaga, B., and K. E. McConnell. 1987. "Exploring Existence Value.” Water Resources Research 23: 936-42.

Mäler, K. G. 1974. Environmental Economics: A Theoretical Inquiry. Baltimore MD: Johns Hopkins University Press for Resources for the Future.

McConnell, K. E. 1983. "Existence and Bequest Value.” In Managing Air Quality and Scenic Resources at National Parks and Wilderness Areas, R. D. Rowe and L. G. Chestnut, eds. Boulder: Westview Press.

Phaneuf, D. J., and J. A. Herriges. 1999. "Choice Set Definition in a Kuhn-Tucker Model of Recreation Demand.” Marine Resources Economics 14: 343-55.

Phaneuf, D. J., C. L. Kling, and J. A. Herriges. 2000. "Estimation and Welfare Calculations in a Generalized Corner Solution Model with an Application to Recreation Demand." Review of Economics and Statistics 82: 83-92.

Pollak, R. A. and T. J. Wales. 1992. Demand System Specification and Estimation. New York: Oxford University Press.

Randall, A. 1994. “A Difficulty with the Travel Cost Model.” Land Economics 70: 88-96.

Wales, T. J., and A. D. Woodland. 1983. "Estimation of Consumer Demand Systems with Binding Nonnegativity Constraints." Journal of Econometrics, 21: 263-85. 